Vol. 5, No. 2, 2020

\title{
INVESTIGATION OF WIRELESS PULSE OXIMETERS FOR SMARTPHONE-BASED REMOTE MONITORING OF LUNG HEALTH
}

\author{
Anatoliy Melnyk ${ }^{1,2}$, Yuriy Morozov $^{l}$, Bohdan Havanio $^{l}$, Petro Hupalo ${ }^{l}$ \\ ${ }^{1}$ Lviv Polytechnic National University, Lviv, Ukraine \\ ${ }^{2}$ The John Paul II Catholic University of Lublin, Lublin, Poland \\ Authors'email: anatoliy.o.melnyk@lpnu.ua; yuriy.v.morozov@lpnu.ua; \\ bohdan.i.havano@lpnu.ua; gypalo911@gmail.com
}

Submitted on 17.11.2020

(C) Melnyk A., Morozov Y., Havanio B., Hupalo P., 2020

\begin{abstract}
Coronavirus has the greatest effect on the human lungs. This is why lungs of patients with COVID-19 require constant medical monitoring. One of the tools for the lungs condition monitoring is based on the wireless pulse oximeters connected to smartphones. There is an attempt to analyze the types of pulse oximeters, their technical characteristics, principles of operation and basic requirements for these measuring instruments in the article. According to this study, the most effective pulse oximeters for $24 / 7$ monitoring of lung function were selected. Principles of pulse oximeter functioning and mathematical basis of its operation have been described. The criteria for pulse oximeter selection have been determined, and comparative analysis of pulse oximeters with wireless communication has been conducted.
\end{abstract}

Index Terms: hypoxemia, COVID-19, pulse oximetry, oxygen saturation, mobile applications, remote patient monitoring.

\section{INTRODUCTION}

The appliance of mobile technologies is caused by the following prerequisites: high level and dynamics of distribution of mobile devices, stable interest in their use, high technical characteristics, convenience and portability.

A smartphone is almost always there with a person unlike other computing devices. Everyday use of a smartphone makes it possible to integrate into our lives a whole range of new technologies. Using smartphones is an effective way for monitoring, longterm monitoring and control of the main indicators that characterize the state of the human body. Using mobile technologies it is possible to organize work with the system in remote access mode.

Creating a platform for monitoring the condition of the lungs based on wireless oximeters is one of them. In consequence of the active implementation of oximeters by developers in their devices, the process of measuring blood oxygen saturation has become much more affordable and easier for ordinary people, without the need for constant medical supervision.

On the other hand, when the need for constant medical supervision exists, as it is the case with patients with COVID-19, asthma, etc., there is a need to create cyber-physical systems for $24 / 7$ monitoring of lung function [1]. The selection of pulse oximeter is very important to build these cyber-physical systems. There are a number of features and characteristics that should be considered to choose the most appropriate ones. Therefore, in this article there is an attempt to analyze the types of pulse oximeters, their technical characteristics, functional principles and basic requirements for these measuring instruments, based on which to select pulse oximeters for cyber-physical systems for $24 / 7$ monitoring of lung function.

\section{THE RATIONALE OF THE NEED FOR LUNG CONDITION MONITORING BASED ON A SMARTPHONE}

Many people have problems related with breathing. These people generally can be divided into three groups:

1. People with dangerous professions and occupations: climbers, speleologists, plumbers, miners.

2. People with lung diseases: inflammatory diseases (bronchitis, pneumonia), allergic diseases (bronchial asthma).

3. People with dangerous living conditions: stove gas, household gas leak.

All these people need permanent monitoring of the lung conditions. If they are in severe condition or in danger of deteriorating, the doctor should have to monitor the state of their lungs [2], [3]. This issue became especially relevant during the pandemic of the COVID-19 virus, which can lead to the development of respiratory failure, pneumonia and a number of complications [4], [5].

The condition of the lung is assessed by the following parameters:

- blood oxygen level (SpO2 saturation),

- respiration rate.

There are following devices for measuring the parameters of the lungs: pulse oximeter, spirometer, and single-photon emission computed tomography SPECT (in planar and tomographic modes).

Measuring devices that allow you to assess the condition of the lungs and provide monitoring using 
smartphone must satisfy a number of requirements. The main requirement is, obviously, convenience and portability, which allows to measure parameters independently, wherever a person is.

The SPECT method is used only in specialized clinics, because the equipment is stationary and has a significant size.

The use of a spirometer requires from a patient to perform a number of procedures.

Now only pulse oximeters can satisfy these requirements. The pulse oximeter can be built into wearable electronics - bracelets, smart watches, etc. It is simple to use and measurements can be automated.

Due to the fact that the level of oxygen in the blood, measured by a pulse oximeter, is one of the most important indicators of a satisfactory condition of the body, less than the norm of which warns of obvious lung disorders. Pulse oximeters have become the basic devices in cyber-physical systems for monitoring lung function.

\section{FORMULATION OF THE PROBLEM}

Today, a large number of different pulse oximeters have been created and are in common use. But not all of them are suitable for building cyberphysical systems for 24/7 monitoring of lung function. The task of the work is to determine the requirements for pulse oximeters of these systems, and to conduct a comparative analysis of the characteristics of existing pulse oximeters for their compliance with these requirements to determine those that best fit these requirements [6].

\section{IV.FUNCTIONAL PRINCIPLES OF THE PULSE OXIMETER}

Pulse oximeters in medical institutions and $\mathrm{SpO} 2$ sensors on fitness wristbands work on the same principle. But there is a small difference. And it is the location of the sensors. In a classic pulse oximeter, the LED bulbs are on one side and the photodiode is on the opposite side. LEDs emit light that passes through body tissues and enters the photodiode as shown in Typically, such pulse oximeters are worn on the finger or earlobe. That is, the part of the body that can be easily enlightened. Accordingly, for fitness trackers and smart watches, this option is not suitable, as it is impossible to illuminate the wrist [8].

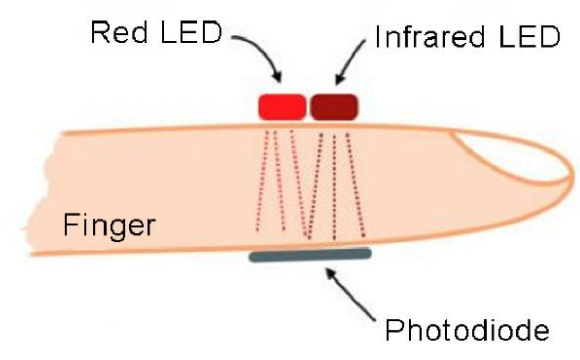

Fig. 1. The passage of light through body tissues
In this case, the photodiode is placed next to the LEDs and the reflected light is analyzed in Fig.2.

In everything else there is no difference. In both cases the same principle is used, the only in medicine the light passed through tissue is analyzed, and in trackers the light reflected from tissue is analyzed. Therewith, there are professional medical pulse oximeters, which work on the same principle as on fitness bracelets.

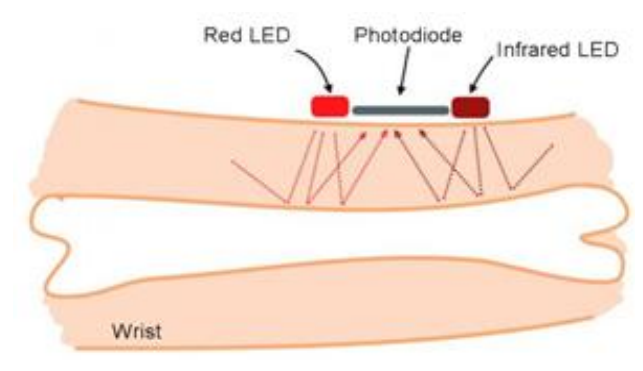

Fig.2. Reflection of light from body tissues

The main question remains - how can simple light calculate the amount of oxyhemoglobin in the blood? And why, by the way, the illustrations show only two LEDs - red and infrared [8]?

There are a few simple facts. First, hemoglobin absorbs light. And secondly, oxyhemoglobin (the one with oxygen) and deoxyhemoglobin (the one without oxygen) absorb light waves of different lengths in different ways.

Now, let's see how light is absorbed by hemoglobin depending on the wavelength (means by color) and type of hemoglobin (oxy- or deoxyhemoglobin) (Fig. 3).

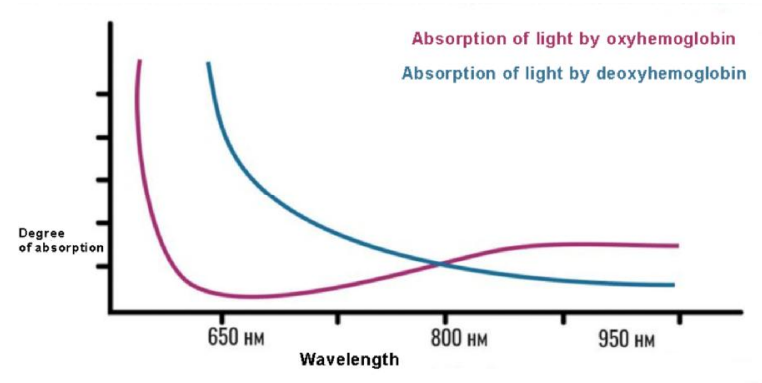

Fig. 3. Absorption of light by hemoglobin depending on the wavelength and type of hemoglobin

As you can see, light with a wavelength of 650 nanometers is practically not absorbed by oxyhemoglobin (with oxygen), but at the same time is maximally absorbed by deoxyhemoglobin (without oxygen). In the figure, respectively, the blue graph at this point $(650 \mathrm{~nm})$ reaches a maximum, and the other $-\mathrm{a}$ minimum.

It turns out that to determine the level of blood oxygen saturation you need to know how much infrared and red light was absorbed by hemoglobin. 
The LEDs light up for a few milliseconds, the light passes through the tissues and arteries, is reflected (mostly from the bone) and returns to the photodiode. Then the computing means of the bracelet compares the amount of light emitted by the LEDs with the amount of reflected light and determines the degree of absorption in the red and infrared spectra.

Of course, this is a very simplified model, designed for ideal conditions that cannot be achieved even theoretically. There are actually many potential problems that need to be solved.

For example, it is clear that not all light will be absorbed by hemoglobin, and the rest will return to the photodiode. A lot of light will be scattered inside the tissues of the body. And the pulse oximeter must distinguish which part of the light was absorbed in the blood and which part was simply scattered.

This problem is being solved by special calibration in the manufacture of devices. And it is that the oxygen level is measured by a pulse oximeter and special laboratory equipment. The person must measure the amount of oxygen in the blood and the indicators are compared. Then a special calibration graph is created, which is used later in the measurements of the bracelet.

There is another, more complex, problem. All people are different. It is one thing to measure on a slender wrist, where the arteries are visible to the naked eye, and quite another - on the wrist of an overweight person. The thicker the skin or adipose tissue, the more light will be absorbed inside the tissues and this has nothing to do with the level of oxygen in the blood.

The solution to this complex problem turned out to be quite simple. You just need to ignore the absorption of light by tissues and analyze only the absorption of light by arterial blood. But how to do it?

During the measurement, all tissues except the arteries will absorb light equally. The fact is that the arterial blood is constantly pulsating and this will affect the amount of light absorbed. After all, the more blood in the artery is, the more light-absorbing material will be. It turns out that when measuring the sensor will constantly see periodic "changes in SpO2", and the values can change every half second (depending on the pulse).

Accordingly, the readings will consist of two data sets: the part that remains unchanged and the part that changes to heart rate. The first data set is useless - it reports only the amount of light absorbed in the tissues, but the second "pulsating" dataset tells us about the saturation of blood with oxygen.

If for some reason the bracelet cannot catch the beat, that is, determine the pulse accurately, it will not be able to accurately determine $\mathrm{SpO} 2$ (oxygen level in the blood).

But, as practice shows, modern fitness trackers can determine the level of oxygen saturation of the blood extremely accurately. However, they do their best when the user is at rest, as any movement creates serious interference in the signal.

\section{MATHEMATICAL BASIS OF PULSE OXIMETER OPERATION}

Pulse oximeter performs mathematical calculations based on the Beer-Lambert-Bouguer law to determine the percentage of oxygen saturation in the blood

$$
I=I_{0} e^{\alpha(\lambda) c d},
$$

where I-intensity of light emanating from the medium, Io - intensity of incident light entering the medium, $\alpha$ $(\lambda)$ - absorption coefficient, which depends on the wavelength, $d$ - path length to be traveled, $c$ - analyte concentration. The properties of the law are valid even if more than one substance absorbs light in the medium.

The total amount of light attenuation can be described by four different components of absorption:

1. Oxyhemoglobin in the blood (concentration of $C_{\mathrm{Hbo}_{2}}$, molar absorption coefficient $\alpha_{\mathrm{Hbo}_{2}}(\lambda)$ and effective path length $d_{\mathrm{Hbo}_{2}}$ )

2. Deoxyhemoglobin in the blood (concentration of $c_{H b}$, molar absorption coefficient $\alpha_{H b}(\lambda)$ and effective path length $d_{H b}$ )

3. Specific absorptions that do not come from arterial blood (concentration of $c_{x}$, molar absorption coefficient $\alpha_{x}(\lambda)$ and effective path length $d_{x}$ )

4. And all other non-specific sources of optical attenuation combined in $A_{0}$, which may include light scattering, geometric factors and characteristics of the generator and detector elements.

Each absorbing element contributes its share to the total absorption

$$
A=\ln \left(\begin{array}{c}
I \\
I_{0}
\end{array}\right)=\alpha_{\mathrm{HbO}_{2}}(\lambda) d_{\mathrm{HbO}_{2}}+\alpha_{H b}(\lambda) c_{H b} d_{H b}+\alpha_{x}(\lambda) c_{x} d_{x},
$$

To calculate oxygen saturation, we first need to calculate the ratio of ripple to constant proportions at different wavelengths:

$$
R=\frac{A C_{R E D} / D C_{R E D}}{A C_{I R} / D C_{I C}},
$$

where $A C_{R E D}$ and $A C_{I R}-\mathrm{AC}$ components of the pulsation of the red and infrared light sources, and a $D C_{R E D}$ and - DC components of the constant red and infrared light sources, respectively.

In practice, the clinical empirical formula for determining the proportion of oxygen saturation is as follows:

$$
S=a-b R
$$

where $a$ and $b$ are coefficients determined when the pulse oximeter is calibrating.[9] 


\section{SELECTING CRITERIA FOR PULSE OXIMETERS}

Pulse oximeters, which are used in cyberphysical systems for 24/7 monitoring of lung function, must be connected to a smartphone via the Bluetooth interface to transmit measurement data.

Mobile applications connected to heart rate monitors must integrate with GoogleFit on Android and HealthKit on iOS to combine data from different sensors.

Pulse oximeters must be portable and have a long battery life.

The accuracy of their blood oxygen saturation measurement should not be lower than in calibrated medical devices.

Pulse oximeters should measure blood oxygen saturation with $\mathrm{SpO} 2$ over certain time intervals without the human intervention.

Based on the above, we can form the following criteria for comparison and selection of pulse oximeters:

- Price,

- Portability,
- Size and weight,

- Wireless communication function with a smartphone (via Bluetooth),

- Ability to export blood oxygen saturation data to GoogleFit on Android and HealthKit on iOS,

- Measurement accuracy,

- Duration of autonomous work,

- Periodic measurement.

\section{COMPARATIVE ANALYSIS OF PULSE OXIMETERS WITH WIRELESS COMMUNICATION}

Based on the conducted analysis of pulse oximeters available on the market, devices that can be used in cyber-physical systems for 24/7 monitoring of lung functions were selected, what means they are portable, have wireless communication function with a smartphone (via Bluetooth) and provide the ability to export saturation data blood oxygen $\mathrm{SpO} 2$ to GoogleFit on Android and HealthKit on iOS. Data on their compliance with the above criteria are summarized in Table 1.

Table

\section{Characteristics of pulse oximeters}

\begin{tabular}{|c|c|c|c|c|c|c|c|}
\hline Name and description & Appearance & Price & $\begin{array}{c}\text { Measure } \\
\text { ment } \\
\text { accuracy }\end{array}$ & $\begin{array}{c}\text { Periodic } \\
\text { measurement }\end{array}$ & $\begin{array}{c}\text { Battery } \\
\text { life }\end{array}$ & $\begin{array}{l}\text { Data export, } \\
\text { Google or } \\
\text { Apple }\end{array}$ & $\begin{array}{c}\text { Dimensions } \\
, \mathrm{cm} / \\
\text { Weight, } \mathrm{g}\end{array}$ \\
\hline 1 & 2 & 3 & 4 & 5 & 6 & 7 & 8 \\
\hline $\begin{array}{l}\text { Pulse oximeter } \\
\text { BM1000A.[10] } \\
\text { Pocket, for measurements } \\
\text { on a finger. }\end{array}$ & & 4800 & $98 \%$ & Manual & $\begin{array}{l}\text { Depends } \\
\text { on } \\
\text { batteries }\end{array}$ & + & $70 \mathrm{~g}$ \\
\hline $\begin{array}{l}\text { Pulse oximeter } \\
\text { "BIOMED" BP- } \\
\text { 10VB[11] } \\
\text { For measurements on the } \\
\text { finger. }\end{array}$ & & 1185 & $98 \%$ & Manual & \begin{tabular}{|l|} 
Depends \\
on \\
batteries
\end{tabular} & + & $50 \mathrm{~g}$ \\
\hline $\begin{array}{l}\text { Beurer PO } 60 \text { Bluetooth }{ }^{\circledR} \\
\text { pulse oximeter[12] } \\
\text { For measurements on the } \\
\text { finger. }\end{array}$ & & $\sim 3000$ & $98 \%$ & Manual & \begin{tabular}{|l|} 
Depends \\
on \\
batteries
\end{tabular} & + & $\begin{array}{l}58.5 \times 30 \\
33 \\
49 \mathrm{~g}\end{array}$ \\
\hline $\begin{array}{l}\text { Pulse oximeter Xiaomi } \\
\text { iHealth Air Wireless } \\
\text { Pulse Oximeter PO3[13] } \\
\text { For measurements on the } \\
\text { finger. }\end{array}$ & & 1049 & $98 \%$ & Manual & \begin{tabular}{|l|} 
Depends \\
on \\
batteries
\end{tabular} & + & $\begin{array}{lll}62 \times 33 \\
28, & & \\
50 \mathrm{~g} & & \end{array}$ \\
\hline
\end{tabular}


Contunuated of Table

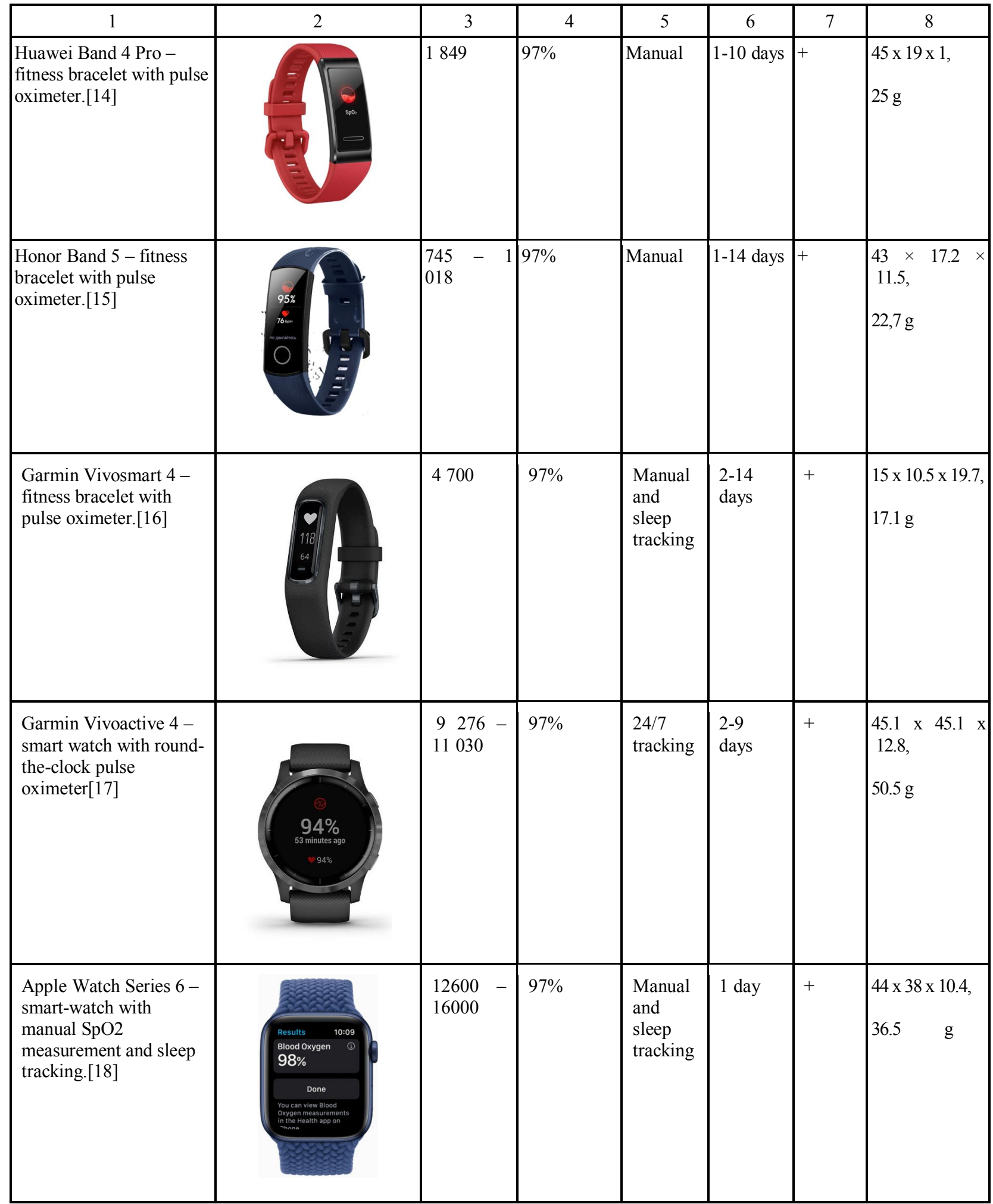

\section{CONCLUSIONS}

By 2020, the creation of a platform for monitoring the condition of patients' lungs based on wireless oximeters is possible and necessary. Thanks to the active oximeters implementation by developers in their devices, the process of measuring blood oxygen levels has become much more accessible and easier for ordinary people.

The types of pulse oximeters, their technical characteristics, principles of operation and basic requirements for these measuring instruments, according to which to select pulse oximeters for cyber-physical 
systems for $24 / 7$ monitoring of lung function were analyzed in the article.

Patients need constant monitoring of the lungs condition, and if they are in serious condition or at risk of deterioration, the doctor should monitor the condition of the lungs. This issue became especially relevant during the pandemic of the COVID-19 virus, which can lead to the development of respiratory failure, pneumonia and a number of complications.

Based on the conducted analysis of pulse oximeters available on the market, devices that can be used in cyber-physical systems for 24/7 monitoring of lung functions were selected. From the point of view of ease of use, fitness bracelets are the best, because they do not interfere with daily human activities. In terms of frequency of measurements, the best are smart watches and pulse oximeter on finger - Beurer PO 60. In terms of autonomy, bracelets and watches are the best. In terms of measurement accuracy, all tested oximeters are approximately equal. Smartphones are the leaders in terms of compatibility with services.

That's why smartwatches are the best for $24 / 7$ monitoring of lung function in cyber physics systems according to a set of criteria. They exceed other tested pulse oximeters in all criteria except for ease of use, where they are second only to fitness bracelets.

\section{ACKNOWLEDGMENTS}

The results presented in this paper were partially obtained within the framework of the project "Applications mobiles pour la surveillance 24h /24 de la fonction pulmonaire chez les patients atteints de COVID-19', funded by the L'Agence universitaire de la Francophonie AUF.

\section{REFERENCES}

[1] Shrikrushna Subhash Unhale, Quazi Bilal Ansar, Shubham Sanap, Suraj Thakhre, Shreya Wadatkar, Rohit Bairagi, Suraj Sagrule and K. R. Biyani. A REVIEW ON CORONA VIRUS (COVID-19). International Journal of Pharmaceutical and Life Sciences, April 2020, Vol. 6, Issue 4, 109-115.

[2] Dai H, Zhang X, Xia J, Zhang T, Shang Y, Huang R, et al. High-resolution chest CT features and clinical characteristics of patients infected with COVID-19 in Jiangsu, China. Int J Infect Dis IJID Off Publ Int Soc Infect Dis. (2020) 95:106-12. doi: 10.1016/j.ijid.2020.04.003.

[3] Andrew M. Luks, and Erik R. Swenson. Pulse Oximetry for Monitoring Patients with COVID-19 at Home: Potential Pitfalls and Practical Guidance. ANNALSATS Articles in Press. Published June 10, 2020 as 10.1513/AnnalsATS.202005-418FR Copyright (C) 2020 by the American Thoracic Society.
[4] World Health Organization. Novel coronavirus (2019nCoV) situation report - 13. 2020, [cited 2020 Mar 4]. Available from:

[5] https://www.who.int/docs/defaultsource/coronaviruse/situation-reports/20200202-sitrep13-ncov-v3.pdf?sfvrsn=195f4010_6.

[6] Giuseppe Di Lorenzo and Rossella Di Trolio. Coronavirus Disease (COVID-19) in Italy: Analysis of Risk Factors and Proposed Remedial Measures. Front. Med., 09 April 2020 | https://doi.org/10.3389/fmed.2020.00140.

[7] O'Carroll O, MacCann R, O'Reilly A, et al. Remote Monitoring of Oxygen Saturation in Individuals with COVID-19 Pneumonia. Eur Respir J 2020; in press (https://doi.org/10.1183/13993003.01492-2020).

[8] Andrew M Luks, and Erik R. Swenson. Pulse Oximetry for Monitoring Patients with COVID-19 at Home: Potential Pitfalls and Practical Guidance. ANNALSATS Articles in Press. Published June 10, 20оксим20 as 10.1513/AnnalsATS.202005-418FR.

[9] Rusyn V.I. Characteristics not invasive method of measuring pulse and blood and saturation and device algorithm. CSM, 2015. Available here: http://eom.lp.edu.ua/ sntk/2015.html

[10] How pulseoximeter works. Available from:

[11] https://deep-review.com/articles/how-pulse-oximetersworks-on-fitness-trackers

[12] Pulse oximeter BM1000A

[13] https://www.shberrymed.com/handheld-pulseoximeter-p00011p1.html

[14] Pulse oximeter "BIOMED" BP-10VB

[15] https://zakupka.com/p/867136170-pulsoksimetrbiomed-vr-10vb-s-bluetooth-4-0

[16] Beurer PO 60 Bluetooth ${ }^{\circledR}$ pulse oximeter

[17] https://www.beurer.com/web/gb/products/medical/ecgand-pulse-oximeter/pulse-oximeter/po-60bluetooth.php

[18] Pulse oximeter Xiaomi iHealth Air Wireless Pulse Oximeter $\mathrm{PO} 3$

[19] https://www.xiaomi.ua/tonometer/pulsoksimetrihealth-air-wireless-pulse-oximeter-po3/

[20] Huawei Band 4 Pro - fitness bracelet with pulse oximeter.

[21] https://consumer.huawei.com/ua/wearables/band4-pro/

[22] Honor Band 5 - fitness bracelet with pulse oximeter.

[23] https://www.hihonor.com/global/products/wearables/h onorband5/

[24] Garmin Vivosmart 4 - fitness bracelet with pulse oximeter.

[25] https://buy.garmin.com/en-US/US/p/605739

[26] Garmin Vivoactive 4 - smart watch with round-the-clock pulse oximeter

[27] https://buy.garmin.com/en-US/US/p/643382

[28] Apple Watch Series 6 - smart-watch with manual SpO2 measurement and sleep tracking.

[29] https://www.apple.com/ru/newsroom/2020/09/applewatch-series-6-delivers-breakthrough-wellness-andfitness-capabilities/ 


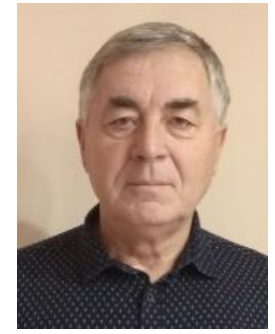

Anatoliy O. Melnyk has been a Head of the Department of Computer Engineering at Lviv Polytechnic National University since 1994 and a Head of the Department of Artificial Intelligence of John Paul II Catholic University of Lublin since 2018. He graduated from Lviv Polytechnic Institute with the Engineer Degree in Computer Engineering in 1978. In 1985 he obtained his $\mathrm{PhD}$ degree in Computer Systems from Moscow Power Engineering Institute. In 1992, he received his DSc degree from the Institute of Modeling Problems in Power Engineering of the National Academy of Science of Ukraine. He was recognized for his outstanding contributions to high-performance computer systems design as a Fellow Scientific Researcher in 1988. He became a Professor of Computer Engineering in 1996. From 1982 to 1994 he was a Head of the Department of Signal Processing Systems at Lviv Radio Engineering Research Institute. From 1994 to 2008 he was a Scientific Director of the Institute of Measurement and Computer Technique at Lviv Polytechnic National University. From 1999 to 2009 he was a Dean of the Department of Computer and Information Technologies at the Institute of Business and Perspective Technologies. Since 2000 he has served as President and $\mathrm{CEO}$ of Intron ltd. He was also a professor at Kielce University of Technology, Rzeszow University of Information Technology and Management, a visiting professor at University of Bielsko-Biala.

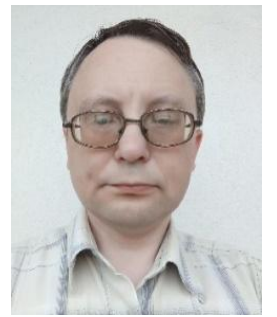

Yurii Morozov was born in 1967 in Lviv, Ukraine. $\mathrm{He}$ received an engineering degree in radio physics and electronics at Lviv State University in 1989 and a Ph.D degree from Lviv Polytechnic National University in 1997 and was awarded the title of associate professor in 2004.. He is currently an associate professor at the Department of Computer Engineering at Lviv Polytechnic National University. His research interests include medical, navigation, travel and data protection in cyber-physical systems.

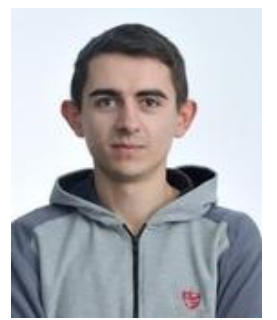

Bohdan Havano was born in 1994 in Sambir, Ukraine. He received the B.S. degree in computer engineering at Lviv Polytechnic National University in 2015 and M.S degree in system programming at Lviv Polytechnic National University in 2016. He has been doing scientific and research work since 2017. Currently, he is a graduate student of the Computer Engineering Department at Lviv Polytechnic National University. His research interests include architecture and data protection in cyber-physical systems.

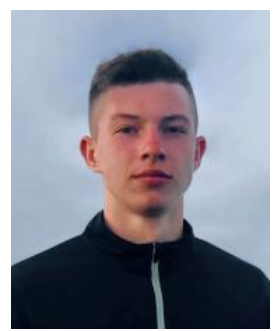

Petro Hupalo was born in 1999 in Lutsk, Ukraine. He is a fourth-year student in computer engineering at Lviv Polytechnic National University. $\mathrm{He}$ was involved in some technological startups. His interests are related to machine learning, data analytics, and forecasting. 